\title{
Gesture Controlled Mouse
}

\author{
https://doi.org/10.3991/ijoe.v13i04.6898 \\ Pallavi \\ SRM University, NCR Campus, India \\ Vinod Kumar \\ SRM University, NCR Campus, India \\ Praveen Kumar \\ SRM University, NCR Campus, India \\ Praveenmps 1994 @gmail.com \\ Anurag Singh \\ SRM University, NCR Campus, India
}

\begin{abstract}
Gesture controlled mouse is a device in which we can move the mouse by giving the direction from our wrist and turn the direction of mouse through it. After using a accelerometer we can able to move and change the direction of our mouse and by the help of accelerometer we can also change the speed of the mouse. This is a new technology for controlling any device through our wrist movement. After upgrading it we can make many type of other devices which can be very useful for the human being can create a turn in our life. It can be used at many places and also be the part of the future. The gesture will be main part as the movements of our body will play the important role for operations. A stationary point will be taken as the directions will be in $\mathrm{x}$-axis, $\mathrm{y}$-axis and as well as the z-axis. An accelerometer will be used as the direction indicators.
\end{abstract}

Keywords-Gesture, Accelerometer

\section{Introduction}

Recently, due to digitization in every field, data processing speeds have increased dramatically, with computers being advanced to the levels where they can assist humans in complex tasks. Yet, input technologies seem to cause a major bottleneck in performing some of the tasks by under-utilizing the available resources and usage restriction as a result of cost constraint. Compared to the optical mouse which offers a limited range of length of connecting cable and also they require a surface to work on, the wireless in not of much use other than allowing for a desktop with fewer wires attached. HUMAN COMPUTING INTERACTION (HCI) is one of the important area of research were people try to improve computer technology. Gesture is a very natural human communication capability. The device is basically a microcontroller 
device which has the PIC 16F877A as the central system. The other components are basically rectifier, filter, transformers, LEDs , capacitors, resistors and Voltage regulator. Here the gestures are recognized by the accelerometer. The directions namely the $\mathrm{X}$ - axis , $\mathrm{Y}$-axis and the $\mathrm{Z}$ - axis are used. The movements of the hands controls the movements of the cursor of the computer.

\section{Gesture Controlled Technique}

Often gestures are in two forms: 1. Static i.e. which may require less computational complexity or 2. Dynamic i.e. which may require or may be more complex but are suitable for the real time environment. As we know are several other methods which have been proposed for the gesture recognisation. But here we are using the hand gesture method for the movement of the cursor without the traditional use of the optical mouse.

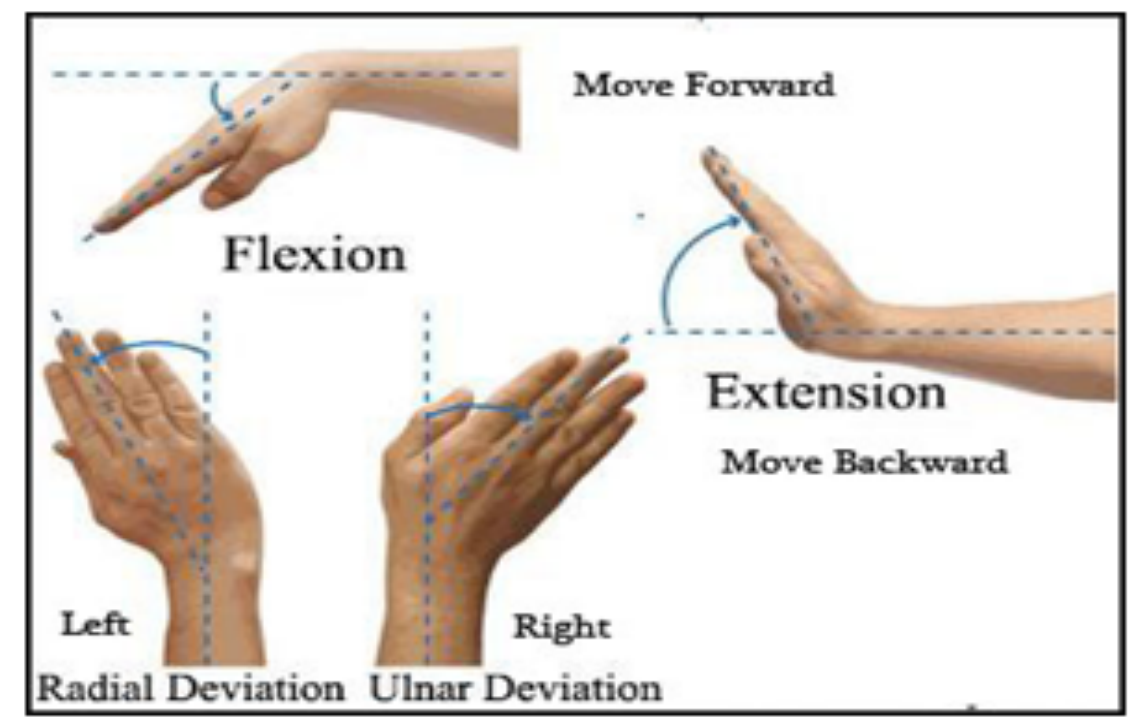

Fig. 1.

The detail about its working is given below.

Step 1:During the stationary position the changes along the $\mathrm{x}, \mathrm{y}$ and $\mathrm{z}$ direction will be zero so during stationary position mouse will not move.

Step 2:During the tilt of our wrist in the up state. The $x$-axis of the accelerometer will change in positive direction so our mouse will start moving backwards.

Step 3: During the tilt our wrist in the down state. The x-axis of the accelerometer will change in negative direction so our mouse will start moving in the forward direction.

Step 4: During the tilt our wrist in the left direction. The y-axis of the accelerometer will be change in negative direction so our mouse will move in the left direction. 
Step 5: During the tilt our wrist in the right direction. The y-axis of the accelerometer will be change in positive direction so our mouse will move in the right direction.

\section{Hardware Implementation}

To interface our project with computers, we realized that we would be required to implement a USB HID class device. During the planning stages of our design, we researched different ways implementing a USB HID and discovered that we could either proceed by doing this through software or hardware support. We came to the conclusion that our design would require a micro-controller in our base station in order to receive wireless messages and process the information. With this in mind, we decided it would be a more practical and simple to purchase a single hardware board that could perform both these functions. So we purchased a micro controller with which we used transmitters for receiving as well as sending. The microcontroller which we are using is the PIC series microcontroller i.e. PIC 16F877A. The other components used are the transistors for sending and receiving signals i.e. BC547. The LCDs and LEDs are also used. The voltage regulator 7805 is also used. The resistors, capacitors, diodes and accelerometer is also used .

\section{Software Implementation}

The software used for this project is Keil C cross compiler as it has a microcontroller. This compiler is basically used for the conversion of the high level language to the object code. The embedded $\mathrm{C}$ language is used for the programs which are given to the microcontroller. The embedded $\mathrm{C}$ language used is for giving instructions.

\section{Application}

Gesture recognition, technology, is achieving rapid market adoption as it evolves and matures. These are based on embedded vision algorithms that use cameras to detect and interpret finger, hand and body movements. However, gesture recognition as a user interface scheme also applies to a wide range of applications beyond consumer electronics. In our project is very useful as we can use our gestures for controlling the cursor of the mouse. In the automotive market, for example, gesture is seen as a convenience-driven add-on feature for controlling the rear hatch and sliding side doors. Cameras already installed in rear of the vehicle for reversing, and in the side mirrors for blind spot warning, can also be employed for these additional capabilities. Gesture interfaces are also useful in rehabilitation situations. Gestures' IREX, for example, guides patients through interactive exercises that target specific body parts. And less conventional health-related applications for gesture recognition also exist. It is also useful in the 3-D modelling and also it can also be the part of the sign language recognisation. 


\section{Advantages}

- Portable

- Cost effective

- Open source software

- Data access directly from the machine to the real time

- Support multi touch and multiple user interaction

- Mind map the idea anywhere.

\section{$7 \quad$ Disadvantages}

- Post processing can occur.

- Hardware limitation on carrying everywhere

- Many phones and laptop doesn't allow to use their camera in the real time space.

\section{$8 \quad$ Costing}

In this project we are using two microcontrollers of PIC series which will cost 400 each thus the total will be of 12 US dollars. The circuit diagram printing on the PCB board will be for both microcontrollers, so for each it will take 1200 , so for both it will be a total of 36.54 US dollars. The components used such as LEDs, capacitors, resistors and other components will cost 200. The cost of the transformer 1.54 US Dollars and transistor is 2.4 US Dollars. For connecting the laptop to the microcontroller we need a TTL USB which will cost us 4-5 US Dollars. The battery is used will costs us approximately 2-3 US Dollars. So the overall total costing for the project will be of 68-70 US Dollars.

\section{Conclusions}

As per the objective a portable embedded device consisting of tri-axial accelerometer, microcontroller (PIC16F877A) and wireless communication module has been built and tested. The acceleration signals measured from the accelerometer are transmitted to the computer via the wireless module. In this project, MEMS accelerometer measures the acceleration of the signal in three co-ordinates such as $\mathrm{x}$-axis, $\mathrm{y}$-axis, and z-axis. An executable file should be installed in PC. A required COM port is selected and enabled. Then moving the accelerometer will also produce the movement of the computer cursor. This system could be useful in presentations and to reduce work space. 


\section{References}

[1] "The PIC Microcontroller and Embedded systems" by Muhammad Ali Mazidi and Janice Gillespie Mazidi , Pearson Education.

[2] PIC16F877A Data Sheets.

[3] www.microchip.com

[4] www.beyondlogic.org

[5] www.wikipedia.org

[6] www.howstuffworks.com

\section{Authors}

Pallavi is student at the Department of Electronics and Communication Engineering, SRM University, NCR Campus, India.

Vinod Kumar is student at the Department of Electronics and Communication Engineering, SRM University, NCR Campus, India.

Praveen Kumar (corresponding author) is student at the Department of Electronics and Communication Engineering, SRM University, NCR Campus, India (Praveenmps 1994 @gmail.com)

Anurag Singh is Assistant Professor at the Department of Electronics and Communication Engineering, SRM University, NCR Campus, India.

Article submitted 16 March 2017. Published as resubmitted by the authors 23 April 2017. 